\title{
AN INVESTIG ATION IN TO APPLICATION OF DECISION MAKING MODELS FOR CREDIT SCORING IN A STATE SECTOR BANK
}

\author{
Vindu Palihakkara* \\ School of Computing - Asia Pacific Institute of Information Technology \\ Colombo, Sri Lanka \\ E-mail: vindu87@gmail.com \\ Manesha Peiris \\ School of Computing - Asia Pacific Institute of Information Technology \\ Colombo, Sri Lanka \\ Email: manesha@apiit.lk
}

\begin{abstract}
Credit scoring is a vital activity conducted by financial institutes which involves a discrete decision making process in which the loan is assessed to avoid financial risk. However this process is often biased as it involves a degree of personal preference due to the unavailability of suitable decision making models. Objectives of research conducted was to identify a decision making model for banks to evaluate credit risk in granting loans; thereby reducing other risks. In order to achieve this objective a careful scrutiny of related papers was conducted looking into weight-age models such as Analytical Hierarchy Process, Multi-Attribute Utility Theory, Decision Tress, Logistic Regression and Artificial Neural Networks. This research highlights application of a hybrid model utilizing Analytical Hierarchy Process (AHP) in combination with decision trees in order to solve the problem. Decision trees were used as the foundation for the basic loan approval process. While AHP was used to develop a weighting model that follows pairwise comparison identifying the relative importance of different criteria identified in loan applications, backing up the Decision Tree while improving accuracy rates. The proposed model proved accurate and timely decisions while increasing throughput efficiency by $29 \%$ and reducing manual labour by $54 \%$ compared to the current manual process.
\end{abstract}

Keywords: Decision Trees, Analytic Hierarchy Process

\section{Introduction}

With the end of war crisis in Sri Lanka, banking and finance industry is now booming. New constructions in the north and east and low income rates in the country are few major reasons for this. As a result general public seek help from banks for housing constructions. Currently the total number of loans granted by commercial banks in Sri Lanka is at a rapid increase and the annual demand for purchasing or building houses is 100000 , out of which $80 \%$ are private constructions (SMIB, 2007).

Though above facts prove that the social environment is in favour for banks, current loan approval process carried out by SMIB is often biased due to personal preferences. This leads to high financial risks, time lag between different stages of the process, waste of resources (time and labour) resulting in customer dissatisfaction leading to less competitive advantage in the industry, despite the favourable market.

\footnotetext{
* Corresponding author
} 


\section{Current credit scoring/loan approval process and its drawbacks}

The current credit scoring process involves a considerable number of steps. This leads to time waste and customer dissatisfaction. The decision making process involved in loan approval is also affected by the said manual process. It is often based on personal preferences, lacks transparency and is biased. Therefore increases credit risk. The process also involves a large number of labour and other resources such as stationary, office space etc. which are additional costs for the bank. In order to prove the inefficiency of this process mathematically, throughput efficiency was calculated.

Table 1: Throughput effic iency of the current process

\begin{tabular}{|l|l|l|l|l|l|l|}
\hline Process & $\begin{array}{l}\text { Applica- } \\
\text { tion }\end{array}$ & $\begin{array}{l}\text { File genera- } \\
\text { tion }\end{array}$ & Valuation & Approval & $\begin{array}{l}\text { Legal proc- } \\
\text { ess }\end{array}$ & Disbursement \\
\hline $\begin{array}{l}\text { Throughput } \\
\text { Efficiency (\%) }\end{array}$ & 18.08 & 75.00 & 34.88 & 00.04 & 00.39 & 02.48 \\
\hline
\end{tabular}

Considering above facts, the root cause for de lay in loan approval and credit risk, is the current manual loan approval process. This results in reduction of quality of service provided by SMIB thus reducing its competitive power in the industry.

\section{Problem statement}

A manual loan assessment and approving system can lead to wastage of resources, increase the number of errors, and duplication of work, leading to employee and customer dissatisfaction, reducing churn rate, bringing in less competitive advantage to SMIB.

A solution to overcome above inefficiencies is to identity how to reduce the subjectivity and inefficiencies of the current process whilst improving the quality of decisions made, reduce Non-Performing Loan rate and improving productivity by introducing Decision Support System (DSS) technology. The following section will discuss the application of a DSS framework for the current loan approval process.

\section{Literature review}

\subsection{Cre dit scoring}

According to Crook et al (2007, cited Bastos, 2008, p.1)., Credit scoring is a method which is commonly used in the area of money lending which helps the management of banks identify and assess credit worthiness of a loan applicant. As stated by Wickramaratne (2009 cited Warushamana, 2009) by the end of year 2008 NPL rate was Rs.75 billion where as by the end of February it was shown a growth up to Rs.112 billion. According to statistics received from SMIB, percentage of NPL in the ir organization is also at an increase. Table below illustrates the increase of NPL rate of SMIB (SMIB, 2007):

Table 2. NPL rates of SMIB

\begin{tabular}{|l|l|l|l|l|}
\hline Year & 2002 & 2003 & 2004 & 2005 \\
\hline SMIB NPL rates & $20 \%$ & $28 \%$ & (not given) & $39.5 \%$ \\
\hline
\end{tabular}

Therefore SMIB require more focus on decision making with regard to loan approval to reduce risks such as NPLs which is considered a major loss for SMIB and a weakness. 


\subsection{Decision Support Systems (DSS)}

Today DSSs are being implemented in various contexts of applications to make the decision making process highly transparent and reduce risks. The following sections discuss different Multiple Criteria Decision Making methods that could be used to replace the manual decision making model under discussion.

\subsection{Logistic Regression}

Logistic Regression is a credit scoring model which can be used for loan approval. Out of many decision making models used today, responsive variable is mostly quantitative (Larsen, n.d., p.1). There could be only two out comes through logistic regression, which is either 'yes' or no' (Newton, n.d., p.2). Equation used in logistic regression is: $\log \left(\frac{\rho}{1-\rho}\right)=\beta_{0}+\beta_{1} X_{1}+\beta_{2} X_{2}+\ldots+\beta_{n} X_{n}$.

$P$ is the probability of that $Y=1$ and $X_{1}, X_{2}, \ldots . X_{k}$ are the independent variables and $\beta_{0}, \beta_{1}, \beta_{2}, \ldots \ldots \beta_{k}$ are regression coefficients which are taken off from the data.

The answer gained by logistic regression is a linear combination of the explanatory variables. The answer will then be transformed into probabilities by a logistic function. However as stated by Dufty (2007) logistic regression requires a large set of test data, in order to make a stable decision. He also states that the minimum number of data required for each predictor is 50 . Also Geng $(2006$, p.2) mentions that the error terms may be invalid due to assumptions made. He also states this leads to time consumption and hassle in selecting variables. Also there may be other numeric problems that will be faced while incorporating the said model (Geng, 2006, p.3). Considering above facts it can be said that this technique would not be effective when evaluating credit risk, due to complexity, requirement of large amount of test data as well as the large amount of data required for each predictor and major time consumption.

\subsection{Artificial Neural Networks (ANN)}

ANN is another common model which can be used for credit scoring in the financial industry. Even though ANN has many advantages it also has few drawbacks. Similar to Logistic Regression model, ANN also requires a large set of test data (Turban \& Aronson, 1998 p.674) and it slows down the learning speed, due to its complexity (Rudjer Boskovic Institute, 2001). It does not give explic it knowledge in the form of rules (Rudjer Boskovic Institute, 2001). It has also been stated that the results obtained from an ANN is hard to interpret (Hagen, 2006), this is mainly due to complexity of the model. Therefore considering the proposed solution for the problem under discussion and drawbacks of ANN such as high complexity, requirement of large amount of test data, and slow learning speed; this was also eliminated.

\subsection{Decision Trees (DT)}

DT is another model that can be used for assessing credit risk. It is a rule based classifier, predictive model (Satchidananda \& Simha, 2006, p.2). DT is used for making multiple decisions; thus called multiple stage decision making model (Oakshott, 2001, p.186). DT has advantages such as ease of explanation and learning, ability to handle both categorical and nominal data, comprehension of results and ability to perform well even with a large set of data.

According to a survey carried out in the banking industry of Germany success rates of ANN and logistic regression is $76.4 \%$ and $83.86 \%$ respectively (Islam et al, 2009). This shows that ANN has out performed logistic regression model. On the other hand effectiveness of logistic regression model and DT model were analysed depending on several measures (Satchidananda \& Simha, 2006, p.5). These are percentage correctly classified (PCC), true positive percentage (TP), false positive percentage (FP) and precision. The result shows that the DT model has out performed the logistic regression model in all parameters. Result of the survey is as under (Satchidananda \& Simha, 2006, p.5) (Figure 2): 
Table 3. Comparison of success rates regarding DT and LR

\begin{tabular}{|l|l|l|l|l|}
\hline \multicolumn{5}{|c|}{ Defaulters } \\
\hline & PCC (\%) & TP $\%)$ & FP $(\%)$ & Precision (\%) \\
\hline Decision trees & 92 & 93 & 10 & 90 \\
\hline Logistic reg ression & 83 & 83 & 16 & 83 \\
\hline \multicolumn{7}{|c|}{ Non defaulters } \\
\hline & PCC (\%) & TP $(\%)$ & FP $(\%)$ & Precision (\%) \\
\hline Decision trees & 92 & 90 & 6 & 93 \\
\hline Logistic reg ression & 83 & 84 & 17 & 84 \\
\hline
\end{tabular}

\subsection{Weight-age models}

In order to make decisions more transparent and reduce the rate of personal preference, few prioritizing models were closely analyzed. Such models are AHP (Analytic Hierarchy Process) and Multi-Attribute Utility Theory (MAUT). Following chapters give brief introductions to these models and discuss its advantages, disadvantages and usage.

\subsection{AHP}

AHP is a method of relative measurement with scales of both tangible and intangible criteria which is based on the judgment of experts in the industry (Administrator, 2010). The scenario under discussion includes ranking different decision alternates based on different sub criteria, assigning weights and obtaining a score for each applicant. Thus AHP is useful when making a decision as whether to provide a loan to an applicant or not.

This method is very useful when considering a choice. Few areas which AHP is currently being used are selection of one from many alternatives, forecasting, resource allocation, BPR etc. (Anon, nd, p.7). Advantage of AHP is that it allows capturing both subjective and objective evaluation criteria (RF Evaluation Centres, 2010). It also helps keep evaluation measures consistent throughout the process (RF Evaluation Centres, 2010). Also it allows make decisions in any environment despite of social or other influences (Anon, nd) helping make un-biased and transparent decisions.

\subsection{MAUT}

MAUT is another model used for evaluating many attributes while making decisions. This model is currently used by many organisations for decision making purposes (Schafer, nd, p.1). However a limitation of the model is that it is assumption based and these assumptions may lead to wrong decisions if the assumption itse lf is not accurate and making proper assumptions may require high level of skills and practice (LeeMerkhofer Consulting, 2009). Also Collins et el (2006, p.444) mentioned that MAUT is a model which will go robust if number of criteria increases. Since loan approval considers a large amount of criteria and employees have poor know ledge/skills, it is not prudent to use this method for the said purpose.

\subsection{Justification}

Since year 2000 AHP has been widely used by the banking industry for decision making and has been combined with credit rating tools widely nowadays (Bhattarai \& Yadav, 2009, p.1). Main reason for banks to incorporate AHP was the financial crisis which hit in year 2000 (Bhattarai \& Yadav, 2009, p.5). Though AHP is suitable for risk evaluation it may not be the best for decision making re lated to loan approval. Since provision of a loan is not limited to selection of the best ranking applicants, but could be provided to any number of applicants who are above an average rank. Therefore this model alone cannot be considered as the best for the entire loan approval process. 
On the other hand though this model cannot be used for the entire process there is clear evidence that AHP is used for credit scoring along with other models such as TOPSIS as mentioned by Bhattarai \& Yadav (2009, p.3). Looking at above facts it is best to consider AHP for assigning weights and DT for backing AHP as the foundation for the proposed credit scoring model.

Incorporating AHP and DT is commonly seen in many scenarios. As stated by Brozova (2004, p. 77) research was carried out to identify the best methodology to make decisions when managing farms and a gricultural enterprises, in the Czech Republic, to chose the best alternative. Another instance of using AHP along with DT was analysis of risk in large scale IT based projects. The model was AHP based on DTs (Dey, 2002, p.13).

\section{Implementation}

Several change over management procedures were considered in this scenario. Out of which direct cutover, is the least costly method. Nevertheless the risk of this strategy is rather high. Even though thorough research has been carried out there may be times when the system may crash. In such situations the entire process will freeze. Opportunities available to improve the system further will also be low. Since only after implementing the new system the users will be able to give feedback, and it will be too late to sugge st improvements. Since the employees of the bank do not have much knowledge in computing, they may also not like to see a drastic change in their current processes. Therefore considering above facts the direct cutover method is not the most suited for the proposed application.

Considering the phased operation and pilot operation, even though these two methods allow improvements, a long time frame will be needed for implementation. At the same time users may not adopt to these implementation strategies, since different process will be handled differently, resulting in issues between different departments etc. These two methods are based on user feedback as well. Depending on feedback the systems will prone to changes. Therefore rapid changes may occur. As stated above since computer literacy of employees of the bank is poor to medium, they may not enjoy rapid changes of the system, and they may not give proper feedback either. Therefore pilot operations and phased operation are also not the best changeover strategies for SMIB.

On the other hand a parallel operation is costly, since the manual system as well as the proposed system will have to run simultaneously. This will lead to wastage of recourses as well. However it will let the bank managers identify if both systems give the same output based on similar input. If there is a change they can simply look in to the ir proposed application. This may require major financial input, but in the long run this method is much safe. Since the proposed application is a decision making system for a bank, decisions made should be accurate. Thus the selected system was implemented following Parallel Method.

\section{Conclusion}

Based on research carried out it was identified that a model driven framework incorporated with a weight age model would help eliminate drawbacks of the current credit scoring process. Logistic regression requires a large amount of test data and the minimum number of data required for each predictor is fairly large. The reason for rejecting Logistic regression and ANN was mostly due to the complexity of the model and the fact that the quantity of data required to come up with an accurate response was not available. Taking above facts into consideration DT outperforms Logistic regression and ANN models and has fewer drawbacks when compared.

However DT alone was not able to add transparency to the proposed solution and AHP and MAUT were considered. It was indentified that MAUT is highly assumption based and if one goes wrong the final de- 
cision will be impacted and will also go robust if the number of criteria increases. Therefore AHP was considered for the proposed scenario due to its advantages such as consistency, ability to make decisions despite of social or other influences and transparency. Accordingly AHP was used with DTs to minimize the degree of personal preference in the overall approval, enabling pair wise comparisons of each selection criterion. As a result it was identified that AHP and DT has a significance impact in improving the current process.

The solution was implemented using the Parallel Method considering many advantages such as ability to compare results and less hassle for computer illiterate users, over other implementation techniques. The outcome of implementation was an increment of throughput efficiency of the process by $29 \%$ and reducing manual labour by $54 \%$ compared to the current manual credit scoring process.

The proposed solution also brought in benefits such as transparency in decisions being made, thereby reducing credit risk, quick decision making, improved customer services, reduced wastage of resources, data duplication, improved accuracy and these will lead to their primary objective which is improving customer satisfaction and competitive advantage to SMIB.

\section{REFERENCES}

Administrator (2010) About the Analytic Hierarchy Process. Retrieved February 28, 2011 from http:/www.isahp.org/italy2010/static/about-the-analytic-hierarchy-process

Anon (nd) Analytical Hierarchy Process. Retrieved March 22, 2010 from http://www1.ximb.ac.in/users/notice2000.nsf/c317180a11767f0785256499006b15a3/bc972a4e7a1dd8e86 5256d260013b051/\$FILE/ahp.ppt

Bastos, J.A (2008), Credit Scoring with Boosted Decision Trees. Retrieved September 02, 2009 from: http://mpra.ub.uni-muenchen.de/8156/2/MPRA_paper_8156.pdf

Bhattarai, S \& Yadav, S. R., (2009) AHP Application in Banking: Unfolding Utility in a Situation of Financial Crisis. $\quad$ Retrieved January $18, \quad 2010$ from http://www.superdecisions.com/ saaty/ISAHP2009/Final_Papers/11_Bhattarai_and_Shivjee_Roy_Yadav _REV_FIN.pdf

Brozova, H. (2004) The Analytic Hierarchy Process for the Decision Tree with Multiple Criteria. Retrieved January 19, 2010 from http//www.cazv.cz/attachments/5-Brozova.pdf

Collins et al, (2006), The use of Multi Attribute Utility Theory to determine the Overall Best-in-c lass performer in a benchmarking study, Benchmarking: An International Journal, vol 13 (4). Retrieved January 18, $2010 \quad$ from http://www.emeraldinsight.com/Insight/viewPDF.jsp?contentType=Article \&Filename=htm1/Output/Publi shed/EmeraldFullTextArticle/Pdf/1310130402.pdf

Dey, P.K. (2002) A Project Risk Management: A Combined Analytic Hierarchy Process and Decision Tree Approach. Retrieved January $19, \quad 2010$ from http://www.imamu.edu.sa/Data/abstract/management/pm/Project\%20Risk\%20Management\%20-

$\% 20 \mathrm{~A} \%$ 20Combined $\% 20$ Analytic $\% 20 \mathrm{Hierarchy} \% 20$ Process $\% 20 \mathrm{and} \% 20 \mathrm{Decision} \% 20 \mathrm{Tree} \% 20 \mathrm{Approac}$ h.pdf

Dufty, D. (2007), What is Logistic Regression. Retrieved March 21, 2010 from 
http://www.statgun.com/tutorials/logistic-regression.html

Geng (2006), A Comparison between Logistic Regression to Random Forestsfor Exploring Differences in Risk Factors Associated with Stage at Diagnosis between Black and White Colon Cancer Patients. Retrieved March 21, 2010 from http://etd.library.pitt.edu/ETD/available/etd-04122006102254/unrestricted/realfinalplus_ETD2006.pdf

Islam, M.S., Zhou, L., \& Li, F., (2009), Application of Artificial Intelligence (Artificial Neural Network) to Assess Credit Risk: A Predictive Model for Credit Card Scoring. Retrieved September 21, 2009 from http://www.bth.se/fou/cuppsats.nsf/all/ff848a6ce93d08f2c12575d40025ee96/\$file/Thesis.pdf

Larsen, P.V. (n.d.), Logistic Regression. Retrieved September 21, 2009 from http://statmaster.sdu.dk/courses/st111/module14/module.pdf

LeeMerkhofer Consulting, (2009), Technical Terms Used in Project Portfolio Management. Retrieved March 22, 2010 from http://www.prioritysystem.com/glossarylc.html

Newton, E. (n.d.), Logistic Regression. Retrieved September 21, 2009 from http://ocw.mit.edu/NR/rdonlyres/Sloan-School-of-Management/15-075Applied-

StatisticsSpring2003/8C07CE0F-70BB-4C8F-9A7B-9AD0AF643D71/0/lec15_logistic_regression.pdf

Oakshott, L, A. (2001) Essential Quantitative Methods for Business Management, and Finance, $2^{\text {nd }}$ ed, Palgrave, Network.

Rudjer Boskovic Institute (2001) Neural Networks. Retrieved March 21, 2010 from http://dms.irb.hr/tutoria1/tut_nnets_short.php

Satchidananda, S.S. \& Simha, J.B. (2006), Comparing decision trees with logistic regression for credit

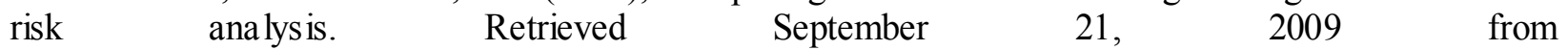
http://www.abibasystems.com/credit_risk_modeling.pdf

Schafer, R., (n.d.) Rules for Multi Attribute Utility Theory for Estimating a User's Interest. Retrieved January $18, \quad 2010 \quad$ from http://www.kbs.unihannover.de/ henze/ABIS_Workshop2001/final/Schaefer_final.pdf

SMIB, (2007), 'Preparation of Corporate Plan 2007-2012' paper presented to the Workshop No.1 of SMIB, Colombo, 4 June.

Turban, E., \& Aronson, J.E., (1998), Decision Support Systems and intelligent systems, $5^{\text {th }}$ ed, Pearson Education, Asia.

Warushamana, G (2009), Global Financial Crisis: Impact on Sri Lanka. Retrieved September 21, 2009 from http://www.sundayobserver.lk/2009/03/29/bus06.asp 